\title{
Lenguas en contacto en Vera de Bidasoa
}

En sus Memorias familiares escribe Caro Baroja: “...el fluir de la vida en Vera era para mí una delicia. Todo me resultaba interesante, atractivo. El paisaje, las personas, el habla de éstas, sus ideas, sus costumbres y los objetos que usaban" ${ }^{1}$. Al releerlas, pensé en la posibilidad de acercarme a Vera a través de los mapas del ALEANR ${ }^{2}$ para estas páginas de homenaje a don Julio.

Observador desde pequeño y partícipe del contacto de lenguas existente en el lugar, su realidad lingüística le era bien conocida. En los años ochenta escribió:

Para cualquier persona que cuando yo era niño llegara a Vera o a un pueblo semejante desde el centro de España, podía ser motivo de extrañeza oír un idioma que no era el castellano. Hoy es menester fijarse más para darse cuenta de que existen personas que hablan vasco. Entonces ocurría lo contrario ${ }^{3}$.

La situación fue cambiando y el vascuence ${ }^{4}$ perdiendo terreno ante el avance inexorable de la lengua de la cultura oficial. Se podía advertir el proceso de generación en generación:

Los niños empezaban a hablar más el castellano y se notaba en las familias diferencia sensible a este respecto entre los mayores y los menores. Mi generación fue todavía de niños que hablaban vascuence. En la de mi hermano en el barrio hablaban mucho menos. Las muchachas de casa lo hablaban entre ellas, y mi abuela se entendía con la que cuidaba la huerta en vasco siempre ${ }^{5}$.

1 Los Baroja. Memorias familiares (con ilustraciones del archivo familiar) (Madrid: Círculo de Lectores, 1986), p. 145.

2 M. Alvar, con la colaboración de A. Llorente, T. Buesa y E. Alvar, Atlas lingüistico y etnográfico de Aragón, Navarra y Rioja, I-XII (Madrid: Inst. Fdo. El Católico-CSIC, 1979-1983).

3 Y añade: "Todo nuestro barrio estaba compuesto de gente que lo hablaba con la excepción de alguna familia de carabinero, que duraba en él una temporada regular como máximo: dos o tres años", en Los Baroja, p. 145.

4Utilizamos vascuence y lengua vasca porque son los términos que prefiere Caro Baroja, aunque hoy estemos relativamente familiarizados con el nombre vasco eusquera.

5 Ibidem. 
Lengua general en Vera, para los Baroja tenía ya un uso desigual entre los distintos miembros de la familia: la usaba más la abuela - los abuelos-, poco los tíos, algo más la madre:

El vascuence en casa no era ya elemento de expresión común. Mis abuelos maternos lo usaron con fluidez. Pero mis tíos no. Menos Ricardo que Pío. Mi madre lo dominaba algo más. De todas formas, la lengua jugaba un papel estético grande para todos, pues eran muchísimas las canciones que también sabían en ella, poseían muchísimo vocabulario ${ }^{6}$.

Además de este papel estético, para don Julio la lengua vasca estaba cargada de valores afectivos, iba de la mano del otoño en Vera:

El recuerdo de las tardes de otoño, cuando se desgranaban las alubias en la cocina y las chicas cantaban canciones viejas del Bidasoa como "Marquesharen alaba" o "Maite bat maitatzaen det" ha quedado grabado en mí de modo indeleble. Así como en Madrid la ola de recuerdos me suele venir con los primeros calores de mayo o junio, en Vera es en el otoño, cuando sopla el viento Sur, o cuando las lluvias de las galernas envuelven "Itzea", cuando siento que se me agolpan. A estas veladas otoñales asocio así mejor que a ninguna otra ocasión mi familiarización con la lengua vasca ${ }^{7}$.

Desde un punto de vista intelectual Caro se preocupó por esa lengua, que también era suya; estudió pronto su retroceso a través de los testimonios históricos y advirtió del interés de volver sobre el enigma que planteaba cuantas veces fuera necesario, desde una postura científica alejada de cualquier apriorismo ${ }^{8}$. De hecho, su propia tesis doctoral ahonda en las profundas relaciones con el latín 9 .

En 1944 publicó en la Biblioteca de Tradiciones Populares del CSIC su monografía etnológica La vida rural en Vera de Bidasoa. Describe en ella minuciosamente la cultura de la villa y, junto a cada nombre castellano, aparece siempre el vasco. Sirvan como ejemplo estas líneas de una de las primeras páginas, en la que recorre la casa típica: "En la planta baja, el portal — "atia"-, la cuadra - "eya"-, el gallinero - "olloteguia" u "ollateguia" - la pocilga — "zerrizteguia" o "ixteguia" - y el estercolero - "gorozteguia" - ${ }^{10}$. El vascuence era por entonces la lengua materna de

6 Ibidem, p. 146.

7 Ibidem.

8 Atlantis, Actas y memorias de la Sociedad Española de Antropología, Etnografía y Prehistoria y Museo Etnológico Nacional, tomo XVI (1941), pp. 31-62.

9 Materiales para el estudio de la lengua vasca en su relación con la latina. (Salamanca: Acta Salmanticensia, 1946).

10 La vida rural en Vera, p. 11. En la Advertencia que precede al trabajo, anota con rigor: "En la transcripción de palabras y textos vascos seguiré dos criterios: 
los habitantes de Vera: "Pasan los años, el niño aprende a hablar en vascuence; luego comienza a ir a la escuela y a aprender algo de castellano" ${ }^{11}$. Vera formaba parte -y sigue haciéndolo- del extremo norte de Navarra, donde se habla vascuence en su dialecto alto navarro septentrional, el de las Cinco Villas ${ }^{12}$.

Cuando en agosto de 1968 Tomás Buesa encuesta en Vera de Bidasoa para el ALEANR, trabaja el cuestionario con un hombre y una mujer, hermanos, de unos 40 años, vascohablantes pero conocedores, a través de la escuela, del castellano, y, por lo tanto, representativos de la situación lingüística de la localidad ${ }^{13}$. En el atlas, Vera es el punto más septentrional de Navarra y en todos los mapas se pueden identificar bajo $\mathrm{Na}$ $100^{14}$ sus respuestas, que suelen ser dobles, en vascuence y en castellano ${ }^{15}$. Buesa explica: "[...] como deseaba reflejar con exactitud la realidad de la localidad explorada, hice simultáneamente dos encuestas, la romance y la éuscara [...] Puede imaginarse la ardua labor que le supuso a un investigador no vascófonon ${ }^{16}$.

$1 .^{\circ}$, cuando se trata de palabras, nombres propios, apellidos, etc., de frecuente empleo en español, se pondrán en ortografía española; $2 .^{\circ}$, lo recogido en la tradición oral, de carácter muy local, en la ortografía que más se ha difundido modernamente" (p. 7).

11 Ibidem, p. 135. En Los Baroja cuenta también, hablando de las muchachas de su casa: "Las chicas eran jovencitas, sacadas del ambiente de Vera, con un castellano problemático y bastante atemorizadas de Madrid" (p. 18).

12 Art. cit., p. 48. Los mapas del ALEANR permiten verlo en su entorno.

13 Las únicas diferencias que se aprecian entre la pronunciación del hombre y la de la mujer se dan en la $/ \Theta /$ castellana: interdental fricativa sorda en la mujer, en el hombre tiende a tener una base de ese dentalizada, como en vascuence. Por lo demás, el castellano presenta los rasgos del de la zona: conserva la diferencia entre $/ \mathrm{l} /$ $1 \mathrm{l}$ y /y/ y, la f-inicial es labiodental, la terminación -ado>-áu, la -d-intervocálica puede ser a veces oclusiva, las vocales finales suelen ser plenas, no relajadas, y se emplea si tendría... por si tuviera... Más información en A. LLORENTE MALDONADO DE GUEVARA, "Las encuestas del "Atlas Lingüístico y Etnográfico de Aragón" y (las encuestas) del "Atlas Lingüístico y Etnográfico de Navarra y Rioja", Archivo de Filología Aragonesa, XVI-XVII, pp. 81-98, especialmente las 96 y 97.

14 También en el Atlas Linguarum Europae (ALE) (Assen: Van Gorcum, 1983-1990) y en el Atlas Linguistique Roman (ALiR) (Roma: Istituto Poligrafico Italiano, 1996) es localidad incluida en la red de puntos, como 017.

15 Castellano es el término que emplean los informantes y, casi siempre refiriéndose a Vera, Caro Baroja. Aquí utilizaremos, también, castellano como sinónimo, aunque desde un punto de vista técnico quizá debiéramos escribir español, ya que en este caso el contacto se da sobre todo con la lengua de instrucción.

16 T. BUESA OLIVER, Unas calas en las hablas de Navarra (Pamplona: Diputación Foral de Navarra, 1980), p. 13. La encuesta en vascuence la hizo sólo en la parte léxica del cuestionario. 
Es sabido que un atlas lingüístico y etnográfico pretende dar una idea de conjunto básicamente de la realidad lingüística, pero también cultural, de una zona. Para ello, a partir de un cuestionario elaborado, recoge los nombres que un mismo concepto recibe en los distintos puntos estudiados y, además del léxico, los rasgos fonéticos, los giros, la morfología; en fin, todo lo que caracteriza esas hablas, de modo que los resultados cartografiados permitan, entre otras cosas, comparar las localidades o trazar límites de fenómenos. La geolingüística de orientación etnográfica unió de siempre palabras y cosas (Wörter und Sachen) y sus atlas reflejan, a través de la lengua, la vida material y espiritual de los hablantes. Un punto queda suficientemente caracterizado con respecto a los demás y, al tiempo, relacionado con su entorno en lo fundamental; pero esa misma dimensión espacial impide, por otra parte, que aparezca tratado con la profundidad de una monografía. De ahí que el estudio de Caro Baroja sobre Vera - tan cuidadoso como el de un buen filólogo cuando nombra lo que describe- y los mapas del ALEANR resulten claramente complementarios. Los mapas nos darán esa visión de Vera ( $\mathrm{Na} \mathrm{100),} \mathrm{del} \mathrm{contacto} \mathrm{de}$ las dos lenguas ${ }^{17}$, y las páginas de don Julio les servirán de apoyo en más de una ocasión.

\section{PRÉSTAMOS}

Desde un primer momento, y aunque conocido, llama la atención el resultado del intenso trasvase histórico desde el latín, que explica algunas de las formas que proporcionaron los informantes de Vera: erregia 'rey' ${ }^{18}$, ferratu 'herrar', gurutzia 'cruz', apilla 'abril', abúztuà 'agosto', abénduà 'diciembre', bastánágria 'zanahoria', kréfala 'trébol', ispilluá 'espejo', nabala

17 Para abordar el tema del contacto entre lenguas desde cualquier ángulo, siempre es básico el libro de U. WeInReich, Languages in contact. Findings and Problems (The Hague: Mouton, 1968). En nuestro caso, especialmente el cap. I. "The Problem of Approach" (pp. 1-5) y el $₫ 2.4$ dedicado a la interferencia léxica (pp. 47-62). Al entregar las pruebas de este artículo, recibo El bilingüismo en el Estado español de M. ETXEBARRIA AROSTEGUI (Bilbao: FBV, 1995), que presta atención documentada al bilingüismo vasco.

18 Los préstamos, que llevan la - $a$ final del artículo, deberíamos traducirlos, de hacerlo literalmente, con artículo: erregia 'el rey', etc. Se ponen acentos sólo para marcar explícitamente dónde recaen. Cuando se hace referencia a conceptos, se mantiene la redonda; la cursiva se reserva para las voces recogidas. Las grafías sólo intentan acercar lo reflejado en transcripción fonética por el encuestador a las formas de los diccionarios vascos. Aunque hemos utilizado mucho el Diccionario vasco-español-francés de R. M. de AZKuE (Bilbao: La Gran Enciclopedia Vasca, 1969), para la grafía final seguimos el Diccionario Vasco-Castellano de Pl. MÚGICA BERRONDO (Bilbao: Ed. Mensajero, 1981) 
'navaja', míspira 'níspero', piper 'pimiento', eliza 'iglesia', garizma 'Cuaresma', besta 'fiesta', komfárdiya 'cofradía', igéltsuà 'yeso', etc., etc., todas ellas sentidas como vascas patrimoniales, junto a la correspondiente voz castellana. Especialmente representativo resulta el caso del vasco lixiba, nombre del agua con ceniza con la que se hacía la colada a la manera tradicional, mientras que lejía corresponde a la actual lejía, y el encuestador explica que ésta, la que se compra, no tiene nombre vasco.

Evidentemente hay que diferenciar entre préstamos históricos desde el latín y préstamos, más o menos recientes en el tiempo, desde el castellano ${ }^{19}$. En la adaptación de los más antiguos se pueden observar procesos conocidos: los que tenían $r$-vibrante múltiple inicial tomaron una vocal epentética: erreka 'reguera', errégiña 'reina', errégluák 'regalos', errégiak 'los Reyes', erramo-eguna 'domingo de Ramos'; la $f$-inicial pudo pasar a $p$-: pikoárbóla 'higuera'; las sordas y las sonoras resultaron inestables en esa posición: bazkua 'la Pascua'; la terminación -ina cambió a -iña: iriña 'harina', kardéliñà 'cardelina, jilguero'. Especialmente en los tomados de una etapa romance, se puede advertir la adición constante de la - $a$ final, que corresponde al artículo, y que no altera mucho los préstamos acabados en consonante: baúla 'baúl', krina 'crín', boza 'voz', jueza 'juez'; pero si la palabra terminaba en $-e$, al añadírsele esa $-a$, pasa a terminar en - $i a$ : frailia 'fraile', tapetia 'tapete', berdia 'verde', pestia 'peste', dotia 'dote', banquetia 'banquete', etc.; del mismo modo, si el final era -o, el resultado era -uà: tráguà, abétua, medíkua, ráguà, latígua. Si la consonante final era $-n$ suele pasar a $-y$, a la que se añade la $-a$ : meloya, pampárroyà 'fanfarrón', melokotoya, porroya, pulmoya, bastoya, si bien se encuentra algún caso como garrafona, urona, saltxitxona. Muchos de los que parecen más recientes sólo se diferencian de su correspondiente voz castellana en la acentuación y suelen ser femeninos en los que la $-a$ del artículo vasco se acopla a la final: maríposà, kampánilla, mantékillà, amápola, margárita, gabárdina, kamiseta, etc.

La vitalidad de los préstamos románicos resulta evidente cuando convive, como vasca, una forma también románica, pero distinta de la que se da como sinónima en castellano: vasc. kandela / cast. vela, koltxilla / sobrecama, mistua / cerilla, danzatu / bailar, kámposántua / cementerio, olídurà / extremaunción. Incluso donde el castellano generalizó un arabismo, aceite, en vascuence podemos encontrar una voz latina, olíyuá, y también el caso contrario: cast. veterinario, pero vasc. albéiteruà. La fuerza del préstamo es tal que entra en la dinámica de la formación de muchas palabras vascas que,

19 Vid. en R. LAPESA, Historia de la lengua española (Madrid: Gredos, 19819), el capítulo dedicado a las lenguas prerromanas, especialmente las pp. 27 y 41. 
en su origen, son mixtas: koltxoiegillia 'pelaire', medikoanandría 'mujer del médico', lañubaju 'nubes bajas', mendípúnta 'cerro cónico', pipérgorriya 'pimiento rojo', sandiúra 'agua, zumo de la sandía', etc.

De los préstamos cabría señalar que los hay para designar determinados productos (aceite, azúcar, harina, mantequilla), sobre todo cuando no son de la tierra (salchichón, derivados de la aceituna, etc.); para el léxico relacionado con la Iglesia (cementerio, entierro, aparecidos, monaguillo, etc.); para partes del ajuar doméstico (armario, espejo, colchón, cuchara, cuchillo); para nombres de cargos en la organización social (rey, maestro, juez, médico, veterinario).

En la terminología agrícola no sólo designan productos más o menos recientes (cacahuete, níspero...), la misma palabra árbolà, que forma la segunda parte de los nombres que se dan a los árboles frutales, es claramente latina: gaztáñarbóla, albárikokearbóla, melókotóyarbola, aránarbóla 'ciruelo', gerézearbóla 'cerezo', gilarbóla 'guindo', sagárrarbóla 'manzano', etc. Los filólogos saben que el préstamo es el resultado habitual del contacto entre lenguas y culturas, pero el abuso de la equivalencia palabra $=$ cosa puede llevar en este aspecto a generalizaciones erróneas: "Una cosa es el origen de la palabra que designa a un instrumento y otra es el origen del instrumento mismo, pero los filólogos parece que no quieren creer esto en muchos casos y piensan que el objeto ha llegado a un país con la palabra" ${ }^{20}$. Desde el latín, los préstamos fueron muchos, como explica el propio Caro: «La influencia directa del latín sobre el vascuence ha sido grande, las palabras que provienen de aquella. lengua son bastante numerosas" ${ }^{21}$; pero merece la pena reproducir aquí su apasionada réplica a Rohlfs ${ }^{22}$, precisamente a propósito de los nombres de frutales:

Algunos han querido dar a entender por esto, o si no han querido lo han hecho de todos modos, que las ideas y cosas que en vascuence tienen nombre latino eran desconocidas por los vascos anteriormente a la romanización del país, romanización siempre relativa. Esto me parece absurdo.

El señor Gerhard Rohlfs de Tubinga ha escrito un ensayo sobre "la influencia latina en la lengua y cultura vascas", ensayo de gran erudición, pero en el que se sigue un criterio tan estrecho que, en ciertos casos, las conclusiones son inadmisibles, como cuando dice que solamente la penetración de los romanos hizo conocer a los vascos los principales árboles frutales, basándose en que la cereza, la castaña, el higo, el membrillo, el melocotón y la níspola tienen nombres procedentes del latín.

$20 \quad$ La vida rural, p. 42.

21 Ibidem.

22 G. ROHFLS, "La influencia latina en la lengua y cultura vascas", Revista Internacional de Estudios Vascos, XXIV (1933), pp. 323-348. 
El señor Rohlfs no nos hará creer que fueron los romanos quienes enseñaron a los vascos a comer castañas. Por otra parte, los otros árboles frutales que hoy día abundan en el país tienen nombre vasco, por ejemplo el manzano, el peral, el ciruelo, el nogal, el avellano y el roble. En cuanto al membrillo y la níspola, no es extraño que tengan nombres de fuera, porque nunca han sido frutos muy propios del país, como los antes citados. Si el señor Rohlfs hubiera dicho que los romanos trajeron algunos árboles frutales, nada se podría objetar a esto; pero decir que hicieron conocer a los vascos los principales es muy diferente ${ }^{23}$.

Páginas después, vuelve sobre el tema al señalar respecto al castaño que: "Es raro el que no tenga un nombre vasco, pero si el árbol no lo tiene, el fruto, o mejor dicho, el erizo, sí, ya que se llama "morkotza" ${ }^{24}$. Y, más adelante, con relación al arado, apela a la existencia de testimonios arqueológicos anteriores a los romanos para afirmar que "aunque la palabra "goldía", que designa al arado, venga del "culter" latino, la etimología no significa nada etnológicamente, lo cual con seguridad debe repetirse con frecuencia" ${ }^{25}$.

En sentido inverso, los mapas del ALEANR dan préstamos del vascuence al castellano en una proporción muchísimo menor. En el habla de los informantes hay voces de origen vasco que se adaptan a su castellano, normalmente en el campo de los juegos y de las voces muy "caseras", cercanas: cast. poner la mía 'poner el badajo' (del vasc. mía 'badajo'), vasc. txarraegúa / cast. charro 'el cerdo más pequeño de la camada', gántxigórrak / ganchigorras 'chicharrones', momflotik / monflotes 'mejillas', tókokà / tocos 'tejuelo', tzurra / zurro 'tacaño', txingokà / ir a chingos 'andar a la pata coja', y parte del léxico del tute, del mus..., aunque no es fácil determinar hasta qué punto se trata de préstamos estables o de adaptaciones del vascuence por interferencia.

\section{EQUILIBRIO DESIGUAL}

Unos informantes aparentemente bilingües, que contestaron preguntas hechas en una de sus dos lenguas, podrían mostrar cierta inclinación por la lengua de encuesta. Resulta clarificador, sin embargo, tratar de ver a través de sus respuestas qué ámbitos léxicos son refugio de la lengua materna y cuáles han sido "invadidos" por la segunda lengua, aprendida, pero potenciada por su papel social.

\footnotetext{
23 La vida rural, pp. 42-43.

24 Ibidem, p. 65.

25 Ibidem, p. 43.
} 
La encuesta en Vera parece haberse desarrollado sin problemas en las dos lenguas: la primera respuesta es la vasca, casi siempre; la segunda, la castellana. Sin embargo, como suele ocurrir cuando se da un contacto estrecho entre lenguas, la materna deja sentir indirectamente su peso sobre la adquirida con una serie de calcos en la segunda lengua que pueden pasar inadvertidos: p.e., el hecho de que el vino turbio se llame sucio se debe a traducción directa de la denominación vasca zikiña, lo mismo que quitar la cabeza es calco de káskuakendú (de kasko 'cráneo' y kendu 'quitar', para 'escamujar'). Casos semejantes son los de culo, como nombre del tocón, lo que queda en tierra de un árbol cuando se corta, y eso porque en vascuence es ipúrdiyà 'trasero, culo'; roble joven para 'quejigo', que reproduce arítzgatzia (de aritz 'roble' y gatze 'joven'); prau de monte para 'alpe', calcado de mendikosorúa (de mendiko 'de monte' y soro 'prado'); cordero de leche para el recental, que podría traducir esnébildotsa (de esne 'leche' y bildots 'cordero que empieza a alimentarse por sí mismo'); perro viejo 'chucho' de zakúrzárra (de zakur 'perro' y zar 'viejo'); ventana ciega, como nombre de la alacena, traslada léioitsúa (de leio 'ventana' e itsu 'ciego'); lo blanco del ojo pone en castellano begíkozuríya (begiko '(lo) del ojo' y zuri 'blanco'), y tantos otros que queden encubiertos ${ }^{26}$.

En otros casos los informantes sólo encontraron la palabra vasca o la palabra castellana: esas presencias o ausencias resultan, entonces, significativas ${ }^{27}$.

Las plantas más corrientes, como el musgo, la ortiga o la amapola, tienen los dos nombres, y lo mismo ocurre con las verduras conocidas, y, en general, con lo que podríamos llamar el léxico básico del cuerpo humano, el de lo relacionado con la infancia, el parentesco, las enfermedades comunes, el cultivo de la vid o el cuidado de las abejas.

A lo largo de la encuesta en ocasiones, pocas, no hay respuesta vasca, y suele ocurrir en cuestiones que tienen al lado el símbolo $\varnothing$, que

26 Sobre el calco como procedimiento de traducción, pueden verse J. P. VINAY y J. DARBELNET, Stylistique comparée du Français et de l'Anglais (Paris: M. Didier, 1973 nouvelle ed. revue et corrigée), pp. 47-48, y, más extensamente, V. García YeBRA, Teoría y práctica de la traducción, vol. I (Madrid: Gredos, 1984²), pp. 341-352.

27 LLORENTE, en su art. cit., p. 96, señala que Julio Fernández Sevilla y él habían atenido como norma recoger las formas vascuences sólo cuando no se conocía la versión castellana (cosa muy frecuente en el vocabulario de plantas, animales silvestres, útiles de la casa, faenas agrícolas y pastoriles, topografía y meteorología) o cuando aun hablando en castellano se usan indistintamente, muchas veces sin tener conciencia el hablante de su distinta filiación lingüística, la forma castellana y la vascuence (no obstante lo dicho, de manera esporádica, pero frecuente se han recogido las formas vascuences aunque no se diera ninguna de las dos circunstancias citadas). 
indica que el concepto por el que se pregunta no existe en la localidad. En estos casos sí se puede decir que es el léxico de lo que no es de la tierra, de lo que no hay o de lo que se conoce a través de la instrucción: útiles de distintos oficios como los nombres del zapapico, de la laya, los de la paleta, la llana, la plomada, la garlopa, etc.; no hay almadías, ni laguna, ni desfiladero, ni guarnicionero, ni tejedor, y pocas monturas de caballo. Muchas enfermedades sólo se conocen por la denominación castellana (asma, sarna, erisipela, varicela) ${ }^{28}$; gran parte del léxico del horno de pan (caja, pala, cajón, brasa, manta); alguna planta y algún pájaro, especialmente si no abundan en el entorno (junco, ruiseñor, pica, alondra, calandria, etc.) y, sobre todo, los productos de huerta que vinieron o vienen de fuera (alcachofa, patata, espinacas, coliflor, granada, lenteja, berengena, acelga, sandía) y algún árbol (llorona 'sauce llorón', alcornoque, algarrobo), junto con nombres de ciertas costumbres (boda, beata, mortaja, cofrade, ángelus, carraca).

En proporción muchísimo mayor ocurre lo contrario, las respuestas vascas son las únicas, lo que evidencia que el bilingüismo sólo existe para el léxico básico y general. Esto es más claro al pasar al más apegado a la tierra, como todo el relacionado con la agricultura -el profesional o específico del informante-tipo. Sólo vascuence para conceptos como barbechar, binar, terciar, la punta de la besana, la amelga, las distintas formas de sembrar, el espantapájaros, escardar a mano, la lleta, el trigo a punto de espigar, la cascarilla, el manojo, la gavilla, el haz, el tresnal, el rastrojo, la hacina, la parva, el corzuelo, etc., etc. Y, aunque en Vera sea poco rico el relacionado con el arado, el léxico de la hierba, el prado y la guadaña resulta completísimo ${ }^{29}$, como ocurre con todo el referido al ganado. Tampoco se conocen las voces castellanas para nombrar partes del cuerpo como la ingle, la corva, la espinilla o los nudillos, ni algunos juegos, ni el trabajo comunitario (auzolán 'azofra', literalmente 'trabajo de vecinos', de auzuá 'vecino' y lana 'trabajo' ${ }^{30}$ ) propio del lugar, ni ciertas vigas y otros elementos de la casa, ni el tronco de Navidad, ni la cuna..., y eso porque no trascienden al mundo exterior en el que se necesitan las dos lenguas.

28 Cf. más abajo y la nota 28.

29 Además de lo que muestran los mapas del ALEANR, en la p. 42 de La vida rural, Caro, apoyándose en los trabajos de Aranzadi, afirma que el vasco «es uno de los países que en menos espacio de tierra ofrece mayor variedad en la tecnología rural y agrícola, dentro de los antiguos tipos". Y eso se refleja en la lengua.

30 Así lo explica Caro en La vida rural, p. 120. Auzolán ocupa gran parte de Navarra, incorporado como voz del castellano de la zona, como se ve en la p. 57 de P. GARCíA MOUTON, "El estudio del léxico en los mapas lingüísticos", Estudios sobre variación lingüistica, recop. F. MORENO FERNÁNDEZ (Univ. de Alcalá de Henares, 1990), pp. 27-75. 
También Caro Baroja, al tratar sobre la medicina popular y sus creencias en Vera, habla de las enfermedades que se conocen, a las que se da nombre:

Las enfermedades que preferentemente tienen nombres vascos exclusivamente suyos, determinados, son las de fácil diagnóstico, es decir, aquellas que se manifiestan por signos exteriores, tales como deformaciones, pústulas, granos, rubicundez, escoriación, etc. Las de la piel sobre todo.

Tienen nombre asimismo afecciones insignificantes, como los callos $\longrightarrow$ babak - y verrugas —kalitxak - , que se manifiestan claramente, y en cambio no lo tienen las grandes enfermedades, la tisis, ni la anemia, el reuma, etc., o si lo tienen, la mayoría de la gente no lo conoce ${ }^{31}$.

Igual que sólo se saben en vascuence los nombres cariñosos para dirigirse al abuelo, sólo se nombran en esta lengua conceptos entrañables de la vida cotidiana como los fenómenos atmosféricos (los vientos, los celajes, los arreboles), las estrellas (el lucero del alba, las tres Marías, la Vía láctea), la flora más cercana y silvestre (el gamón, la retama, el hongo, la zarza, el bojedal, el madroño, el majoleto, la madreselva, el endrino, el peruétano, etc.) o la fauna no doméstica, la de los campos vecinos, cuyos nombres testimonian tantas creencias infantiles (la libélula, el abejorro, el tábano, el ciervo volante, la mariquita, el lución, la salamandra; el petirrojo, la cogujada, el avión, el picamaderos, la aguzanieves, la abubilla, la lechuza, el mochuelo...). La lengua materna sigue dominando estos campos, siempre los mismos, los últimos ${ }^{32}$, como les sucedía también a los Baroja: "...y a veces resultaba que en la conversación surgía una palabra vasca, que no conocíamos en castellano, referente a la flora y a los oficios comunes por lo general" ${ }^{33}$.

Probablemente de entonces a hoy las cosas habrán cambiado en Vera, en ese equilibrio desigual entre vascuence y castellano, pero, incluso entre quienes la hayan perdido como lengua materna, la lengua vasca seguirá presente en esas palabras que siempre se conservan como propias, con afecto y añoranza, como ocurría en casa de los Baroja y recordaba don Julio:

A veces, también, las onomatopeyas vascas, los vocablos que reflejan ciertas acciones, caracteres y rasgos nos parecían más expresivos que los castellanos. De hecho lo son. Para indicar la manera de andar de una vieja ligera es mucho mejor

31 La vida rural, p. 168. La difteria tiene nombre prestado garrótilluà, del cast. garrotillo, y para el concepto de aborto, sólo en vasc. para los animales illora; en cambio, para las mujeres, sólo aborto.

32 Coincide con lo que Llorente señala para otros puntos (vid. nota 25). Lo mismo suele advertirse en la relación entre el léxico dialectal y el de de la lengua general.

33 Los Baroja, p. 146. 
decir que va "zipoca-zipoca" que cualquier otra cosa. Si se quiere dar un matiz a la noción de corcovada es bueno utilizar el vocablo vasco: "curcushada". Si hay que hablar de una ropa vieja o un residuo con caracteres peculiares, la palabra "zerrenda" es magnífica. $Y$ resulta también más exacto e íntimo decir "nere biotza" que "corazón mío. El vascuence aleja de la cursilería y de la altisonancia que pueden tener idiomas literarios más brillantes. Es un idioma íntimo, sutil, con muchos matices humorísticos, pese a las pedanterías huecas que han dicho acerca de él algunos reverendos lingüistas y pensadores. En boca de aldeanos puede ser exactamente lo que es el castellano en boca de gente rústica: o un torpísimo medio de expresión o un idioma sabroso. Cuando lo hablan gentes finas es un idioma fino. Una muchachita hablando vascuence no da casi nunca (con perdón de los que creen que éste es un idioma muy tosco), la sensación de plebeyez que puede dar otra parecida hablando en cualquier habla o patois romance con mucha frecuencia.

Lo que el habla daba a los individuos de las sociedades antiguas era mucho. Cualquiera que haya oído hablar a un viejo campesino de Castilla en forma sentenciosa, llena de modismos, de refranes, con un vocabulario rico puede darse cuenta de esto. Lo que el vasco daba a los hombres y mujeres de otras generaciones es mucho más difícil de pesar y medir. No cabe duda de que rasgos que parecen psicológicos eran, en gran parte, idiomáticos. Formas de humor, de expresión rápida, de imitación. Y.lo mismo entre vascos que entre castellanos no cabe tampoco duda de que las gentes viejas tenían mucho más carácter que las de hoy, con sus ideales cortos y su idioma más corto todavía ${ }^{34}$.

PILAR GARCía MOUTON Instituto de Filología. CSIC

A través de los mapas del ALEANR (Atlas Lingüístico y Etnográfico de Aragón, Navarra y Rioja) y de los escritos de Julio Caro Baroja, se aborda la convivencia de la lengua vasca y el castellano en Vera de Bidasoa. Esta situación se refleja en la presencia de préstamos y calcos entre las dos lenguas. También se estudia qué campos léxicos son más propensos a conservar el léxico patrimonial y cuáles más receptivos a la segunda lengua.

By the use of maps from the Linguistic and Ethnographic Atlas of Aragón, Navarra, and Rioja (ALEANR), as well as Julio Caro Baroja's writings, the article discusses the coexistence of Basque and Castilian in Vera de Bidasoa, which is reflected in the presence of loanwords and translation loanwords from one language to the other. In addition, the article deals with the degree of stability of semantic fields: which ones tend to be more receptive, and which ones more averse, to borrowings from the second language.

34 Ibidem, pp. 146-147. 